Psychological Medicine

cambridge.org/psm

\section{Correspondence}

*These authors contributed equally to this work.

Cite this article: Bai W, Cai $\mathrm{H}$, Jin $\mathrm{Y}$, Zhang $\mathrm{Q}$, Cheung T, Su Z, Tang Y-L, Ng CH, Xiang Y-T (2022). COVID-19 vaccine hesitancy in community-dwelling and hospitalized patients with severe mental illness. Psychological Medicine 1-3. https://doi.org/10.1017/ S0033291721004918

Received: 26 October 2021 Revised: 4 November 2021 Accepted: 8 November 2021

Author for correspondence: Yu-Tao Xiang, E-mail: xyutly@gmail.com

\title{
COVID-19 vaccine hesitancy in community-dwelling and hospitalized patients with severe mental illness
}

\begin{abstract}
Wei Bai ${ }^{1,2,3, \star} \mathbb{D}$, Hong Cai ${ }^{1,2,3, \star}$, Yu Jin ${ }^{4, \star}$, Qinge Zhang ${ }^{5, \star}$, Teris Cheung ${ }^{6}$, Zhaohui Su${ }^{7}$, Yi-Lang Tang ${ }^{8,9}$, Chee H. Ng ${ }^{10}$ and Yu-Tao Xiang ${ }^{1,2,3}$

${ }^{1}$ Unit of Psychiatry, Department of Public Health and Medicinal Administration, \& Institute of Translational Medicine, Faculty of Health Sciences, University of Macau, Macao SAR, China; ${ }^{2}$ Centre for Cognitive and Brain Sciences, University of Macau, Macao SAR, China; ${ }^{3}$ Institute of Advanced Studies in Humanities and Social Sciences, University of Macau, Macao SAR, China; ${ }^{4}$ College of Education for the Future, Beijing Normal University, Beijing, China; ${ }^{5}$ The National Clinical Research Center for Mental Disorders \& Beijing Key Laboratory of Mental Disorders, Beijing Anding Hospital \& the Advanced Innovation Center for Human Brain Protection, Capital Medical University, Beijing, China; ${ }^{6}$ School of Nursing, Hong Kong Polytechnic University, Hong Kong, Hong Kong SAR, China; ${ }^{7}$ Center on Smart and Connected Health Technologies, Mays Cancer Center, School of Nursing, UT Health San Antonio, San Antonio, TX, USA; ${ }^{8}$ Department of Psychiatry and Behavioral Sciences, Emory University, Atlanta, GA, USA; ${ }^{9}$ Atlanta VA Medical Center, Atlanta, GA, USA and ${ }^{10}$ Department of Psychiatry, The Melbourne Clinic and St Vincent's Hospital, University of Melbourne, Richmond, Victoria, Australia
\end{abstract}

A previous study reported that $28.3 \%$ of UK adults were not sure or strongly hesitant to be vaccinated against the COVID-19, and the vaccine hesitancy varies greatly by sociodemographic and clinical background (Freeman et al., 2020). Patients with severe mental illness are more likely to suffer from severe clinical outcomes of COVID-19 (Wang, Xu, \& Volkow, 2021), thus it has been advocated that they should be prioritized for vaccination (De Hert, Mazereel, Detraux, \& Van Assche, 2021). However, vaccine hesitancy in this subpopulation is a major obstacle in the global efforts to control the COVID-19 pandemic. There is little research on this problem apart from two preliminary, single-center surveys conducted in Western countries. A study (Mazereel, Vanbrabant, Desplenter, \& De Hert, 2021) in a Belgium psychiatric hospital $(N=1151)$ found that $93 \%$ of hospitalized patients with various psychiatric disorders accepted and received COVID-19 vaccines. Another study (Danenberg et al., $2021)$ in an Israeli public psychiatric hospital $(N=51)$ found that $23.5 \%$ of hospitalized patients with severe mental illness had no intention to get vaccinated. To date, there are no data on vaccine hesitancy in non-Western settings or in community-dwelling patients with psychiatric disorders or across various diagnostic groups. Therefore, we examined the prevalence of vaccine hesitancy and its associated factors among community-dwelling and hospitalized patients with major depressive disorder (MDD), bipolar disorder (BD), and schizophrenia (SCZ) in China, the largest developing country.

This study was conducted in both outpatient clinics for community-dwelling patients and inpatient departments attached to six major psychiatric hospitals in China between September 21, 2020 and October 8, 2021. Community-dwelling and hospitalized participants who were diagnosed as MDD, BD, or SCZ according to the International Classification of Diseases, Tenth Revision (ICD-10) and older than 18 years were consecutively invited to participate in this survey. Global quality of life was measured with the first two items of the World Health Organization Quality of Life Scale Brief version (WHOQOL-BREF), while severity of perceived fatigue and physical pain were assessed by a fatigue numeric rating scale and Visual Analog Scale for Pain (VAS), respectively. Depressive symptoms were measured by the Patient Health Questionnaire-2 (PHQ-2), and perceived stigma was assessed by the Social Impact Scale (SIS). A standardized question was used to measure COVID-19 vaccination hesitancy: 'Do you intend to be vaccinated against COVID-19 in the future?' (No/Not having vaccination temporarily/Yes). The first two options were categorized as 'COVID-19 vaccination hesitancy' according to the WHO recommendation. All participants signed electronic written informed consent and the study protocol was approved by the ethics committees of the participating hospitals.

The $\chi^{2}$ tests, independent samples $t$ tests, and Mann-Whitney $U$ tests were performed to compare sociodemographic and disorder-related variables between individuals with COVID-19 vaccination hesitancy and those without, as appropriate. Binary logistic analyses were performed to examine independent correlates of COVID-19 vaccination hesitancy, with variables that significantly differed in univariate analyses and primary psychiatric disorders as independent variables. Significantly statistical difference was set at $p<0.05$ (two-tailed).

Altogether, 1853 participants were included in this study. The proportion of COVID-19 vaccination hesitancy was $45.3 \%$ (95\% CI: $43.1-47.5 \%$ ), with $45.3 \%$ (95\% CI: $42.6-48.1 \%$ ) 
Table 1. Characteristics and analyses of participants with and without COVID-19 vaccination hesitancy

\begin{tabular}{|c|c|c|c|c|c|c|c|c|c|}
\hline \multirow[b]{2}{*}{ Variables } & \multicolumn{3}{|c|}{ COVID-19 vaccination hesitancy } & \multicolumn{3}{|c|}{ Univariate analysis } & \multicolumn{3}{|c|}{ Multiple logistic regression } \\
\hline & $\begin{array}{c}\text { All } \\
(N=1853) n(\%)\end{array}$ & $\begin{array}{c}\text { No }(n=1014) \\
n(\%)\end{array}$ & $\begin{array}{c}\text { Yes }(n=839) \\
n(\%)\end{array}$ & $\chi^{2}$ & $\mathrm{df}$ & $p$ & OR & $95 \% \mathrm{Cl}$ & $p$ \\
\hline Male gender & $492(26.6)$ & $260(25.6)$ & $232(27.7)$ & 0.952 & 1 & 0.329 & - & - & - \\
\hline Urban residence & $1310(70.7)$ & $734(72.4)$ & $576(68.7)$ & 3.089 & 1 & 0.079 & - & - & - \\
\hline College education and above & $966(52.1)$ & $543(53.6)$ & $423(50.4)$ & 1.806 & 1 & 0.179 & - & - & - \\
\hline Living with family members & $1477(79.7)$ & $815(80.4)$ & $662(78.9)$ & 0.614 & 1 & 0.433 & - & - & - \\
\hline Married & $665(35.9)$ & $375(37.0)$ & $290(34.6)$ & 1.166 & 1 & 0.280 & - & - & - \\
\hline Unemployed & $1120(60.4)$ & $591(58.3)$ & $529(63.1)$ & 4.364 & 1 & 0.037 & 1.257 & $1.038-1.522$ & 0.019 \\
\hline Having insurance & $1499(80.9)$ & $828(81.7)$ & $671(80.0)$ & 0.839 & 1 & 0.360 & - & - & - \\
\hline Family history of psychiatric disorders & $209(11.3)$ & $104(10.3)$ & $105(12.5)$ & 2.340 & 1 & 0.126 & - & - & - \\
\hline Suicidality in the past year & $1152(62.2)$ & $616(60.7)$ & $536(63.9)$ & 1.920 & 1 & 0.166 & - & - & - \\
\hline Outpatients & $1444(77.9)$ & $733(72.3)$ & $711(84.7)$ & 41.412 & 1 & $<0.001$ & 2.206 & $1.737-2.801$ & $<0.001$ \\
\hline Perceived fair/good health status & $1592(85.9)$ & $877(86.5)$ & $715(85.2)$ & 0.611 & 1 & 0.435 & - & - & - \\
\hline Perceived fair/good economic status & $1476(79.7)$ & $814(80.3)$ & $662(78.9)$ & 0.534 & 1 & 0.465 & - & - & - \\
\hline Diagnoses & & & & 0.926 & 2 & 0.629 & - & - & - \\
\hline MDD & $1214(65.5)$ & $664(65.5)$ & $550(65.6)$ & & & & 1 & - & - \\
\hline $\mathrm{BD}$ & $367(19.8)$ & $207(20.4)$ & $160(19.1)$ & & & & 1.085 & $0.849-1.387$ & 0.514 \\
\hline \multirow[t]{2}{*}{ SCZ } & $272(14.7)$ & $143(14.1)$ & $129(15.4)$ & & & & 1.217 & $0.920-1.612$ & 0.169 \\
\hline & Mean (S.D.) & Mean (S.D.) & Mean (S.D.) & $t / Z$ & df & $p$ & OR & $95 \% \mathrm{Cl}$ & $p$ \\
\hline Age (years) & $30.8(13.4)$ & $31.1(13.4)$ & $30.5(13.3)$ & -1.385 & $-{ }^{\mathrm{a}}$ & 0.166 & - & - & - \\
\hline Age of onset (years) & $27.8(13.6)$ & $28.0(13.3)$ & $27.5(13.9)$ & -1.713 & $-^{\mathrm{a}}$ & 0.087 & - & - & - \\
\hline Fatigue total score & $5.2(2.7)$ & $5.1(2.7)$ & $5.2(2.6)$ & -0.760 & $-{ }^{\mathrm{a}}$ & 0.447 & - & - & - \\
\hline Physical pain total score & $2.9(2.5)$ & $2.8(2.5)$ & $3.0(2.5)$ & -2.507 & $-{ }^{\mathrm{a}}$ & 0.012 & 1.027 & $0.986-1.070$ & 0.196 \\
\hline Global QOL score & $5.2(1.8)$ & $5.3(1.8)$ & $5.1(1.7)$ & -2.915 & $-^{a}$ & 0.004 & - & - & - \\
\hline PHQ-2 total score & $2.8(2.0)$ & $2.7(2.0)$ & $2.9(2.0)$ & -2.191 & $-^{\mathrm{a}}$ & 0.028 & 1.009 & $0.956-1.065$ & 0.756 \\
\hline SIS total score & $62.1(12.1)$ & $61.3(12.1)$ & $63.2(12.0)$ & -3.467 & 1851 & 0.001 & 1.011 & $1.002-1.019$ & 0.011 \\
\hline
\end{tabular}

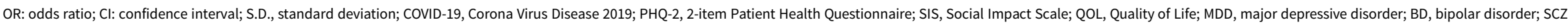
schizophrenia.

${ }^{a}$ Mann-Whitney $U$ test; bolded values: $<0.05$. 
in $\mathrm{MDD}, 43.6 \%$ (95\% CI: $38.6-48.7 \%$ ) in $\mathrm{BD}$, and $47.4 \%$ (95\% CI: 41.6-53.3\%) in SCZ subgroups. The proportion of COVID-19 vaccination hesitancy was $49.2 \%$ (95\% CI: 46.7-51.8\%) in community-dwelling patients, and $31.3 \%$ (95\% CI: 26.9 $35.8 \%$ ) in hospitalized patients. Binary logistical regression analysis revealed that unemployed patients (OR 1.257, 95\% CI: 1.038-1.522, $p=0.019$ ), community-dwelling patients (OR 2.206, 95\% CI: $1.737-2.801, p<0.001)$, and those with a higher level of perceived stigma (OR $1.011,95 \%$ CI: $1.002-1.019, p=0.011$ ) were more likely to have vaccine hesitancy (Table 1). No significant difference in vaccine hesitancy was found across the three major psychiatric disorders.

We found that the vaccine hesitancy rate was high in patients with severe mental illness, particularly in community-dwelling patients, which is considerably higher than the finding in Israel (Danenberg et al., 2021). Consistent with the result in a general population in the USA (Malik, McFadden, Elharake, \& Omer, 2020), there is a higher rate of vaccine hesitancy in those unemployed compared to those employed, which could partly be due to the strong encouragement from all employers in China for vaccination in their employees in response to the pandemic.

Community-dwelling patients with severe mental illness had a higher rate of vaccine hesitancy than hospitalized patients, probably because they received less public education on the importance and safety of the vaccines compared to hospitalized patients. Additionally, hospitalized patients are more likely to experience crowded living conditions compared to their community-dwelling counterparts (e.g. shared common dining and bathroom spaces, and group activities that increase close contacts) (Brody et al., 2021), which may lead to greater awareness of the need to protect themselves. We also found that patients with a higher level of perceived stigma were more likely to have vaccine hesitancy. Psychiatric patients with a higher level of stigma usually have more limited medical knowledge (Thornicroft et al., 2016), and a lack of awareness about the efficacy and safety of COVID-19 vaccination.

In conclusion, this was the first multicenter study on COVID-19 vaccine hesitancy involving both community-dwelling and hospitalized patients with severe mental illness. Due to the high rate of vaccine hesitancy, vaccination promotion strategy should focus on patients with severe mental illness, particularly those who are unemployed and community-dwelling, or have a high level of perceived stigma.

Acknowledgements. The authors thank all clinicians and patients involved in this study.

Conflict of interest. None.

\section{References}

Brody, B. D., Shi, Z., Shaffer, C., Eden, D., Wyka, K., Alexopoulos, G. S., ... Kanellopoulos, D. (2021). COVID-19 infection rates in patients referred for psychiatric admission during a regional surge: The case for universal testing. Psychiatry Research 298, 113833. doi: 10.1016/ j.psychres.2021.113833

Danenberg, R., Shemesh, S., Tzur Bitan, D., Maoz, H., Saker, T., Dror, C., ... Bloch, Y. (2021). Attitudes of patients with severe mental illness towards COVID-19 vaccinations: A preliminary report from a public psychiatric hospital. Journal of Psychiatric Research 143, 16-20. doi: 10.1016/ j.jpsychires.2021.08.020

De Hert, M., Mazereel, V., Detraux, J., \& Van Assche, K. (2021). Prioritizing COVID-19 vaccination for people with severe mental illness. World Psychiatry 20, 54-55. doi: 10.1002/wps.20826

Freeman, D., Loe, B. S., Chadwick, A., Vaccari, C., Waite, F., Rosebrock, L., ... Lambe, S. (2020). COVID-19 vaccine hesitancy in the UK: The Oxford coronavirus explanations, attitudes, and narratives survey (Oceans) II. Psychological Medicine, 1-15. doi: 10.1017/s0033291720005188

Malik, A. A., McFadden, S. M., Elharake, J., \& Omer, S. B. (2020). Determinants of COVID-19 vaccine acceptance in the US. EClinicalMedicine 26, 100495. doi: 10.1016/j.eclinm.2020.100495

Mazereel, V., Vanbrabant, T., Desplenter, F., \& De Hert, M. (2021). COVID-19 vaccine uptake in patients with psychiatric disorders admitted to or residing in a university psychiatric hospital. The Lancet Psychiatry 8, 860-861. doi: 10.1016/s2215-0366(21)00301-1

Thornicroft, G., Mehta, N., Clement, S., Evans-Lacko, S., Doherty, M., Rose, D., ... Henderson, C. (2016). Evidence for effective interventions to reduce mental-health-related stigma and discrimination. The Lancet 387, 11231132. doi: 10.1016/s0140-6736(15)00298-6

Wang, Q., Xu, R., \& Volkow, N. D. (2021). Increased risk of COVID-19 infection and mortality in people with mental disorders: Analysis from electronic health records in the United States. World Psychiatry 20, 124-130. doi: $10.1002 /$ wps.20806 